\title{
Case Report \\ Recurrent abdominal pain in a child caused by a mesenteric lymphatic malformation
}

\author{
Ravibindu Ranawaka \\ Lady Ridgeway Hospital for Children, Sri Lanka,
}

Keywords: mesenteric lymphatic malformation in children, recurrent abdominal pain in children, surgical causes of non-bilious vomiting

Corresponding Author: Ravibindu Ranawaka, E-mail: <rravibindu@yahoo.com > (iD https://orcid.org/0000-0001-9150-7904 Received: November 2018, Accepted: November 2018, Published: December 2018

Competing Interests: Authors have declared that no competing interests exist

(c) Authors. This is an open-access article distributed under a Creative Commons Attribution-

Share Alike 4.0 International License (CC BY-SA 4.0), which permits unrestricted use,

distribution, and reproduction in any medium, provided the original author and source are

attributed and materials are shared under the same license.

\section{Introduction}

Recurrent episodes of abdominal pain associated with non-bilious vomiting in children are often attributed to functional causes or to constipation. In atypical clinical presentations pathologies of surgical origin need to be considered. Relatively common surgical pathologies include; urolithiasis, pelvi-ureteric junction obstruction and partial membranous duodenal obstruction.

An otherwise healthy boy was admitted with progressively worsening episodic central abdominal pain associated with non- bilious vomiting of several months' duration. His pain always appeared after meals and this suggested the pathology being related to the intestines. Intestinal luminal obstruction, however, induces bilious vomiting, and therefore the aetiology of his pain was difficult to determine. He had had many radiological and haematological investigations that were within the normal range.

At diagnostic mini-laparotomy, a lymphatic malformation, confined to the small bowel mesentery, which distends after meals causing compression of the mesenteric vessels and inducing ischaemic pain was identified. Abdominal lymphatic malformations presenting in this manner in children, is extremely rare, according to the literature $[1,2,3]$.

\section{Case presentation}

A nine-year-old boy was admitted with an episode of severe abdominal pain and nonbilious vomiting. He had had many such episodes in the past and had undergone multiple abdominal x-rays and ultrasound scans, been subjected to contrast imaging of the upper gastro-intestinal tract twice and a lower gastro-intestinal contrast study once with normal results. Multiple hematological and biochemical investigations were normal. 
He was kept under close observation to study the pattern of abdominal pain and to look for any positive clinical features. The pain always appeared around half to one hour after ingestion of a heavy meal and resolved only with the use of potent analgesics such as pethidine. After about an hour the pain usually subsided spontaneously. Non-bilious vomiting occurred at the height of the pain and was small in volume.

An upper gastro-intestinal double contrast computed tomography (CT) scan was performed and reviewed by a panel of expert radiologists but no pathology was identified. Magnetic resonance imaging (MRI) scan and laparoscopy were not available. A multidisciplinary team discussion with paediatricians was inconclusive. Finally, a diagnostic mini laparotomy was performed via a standard right upper quadrant transverse incision [4].

A lymphatic malformation of around $30 \mathrm{~cm}$ in length, arising from the ileal mesentery extending up to approximately $35 \mathrm{~cm}$ from the ileo-caecal junction was found (Figure 1). The rest of the abdominal viscera were normal. The mini-laparotomy was converted to a standard laparotomy and the segment of mesentery together with the small bowel was resected and sent for histology. End to end anastomosis of the healthy bowel was performed.

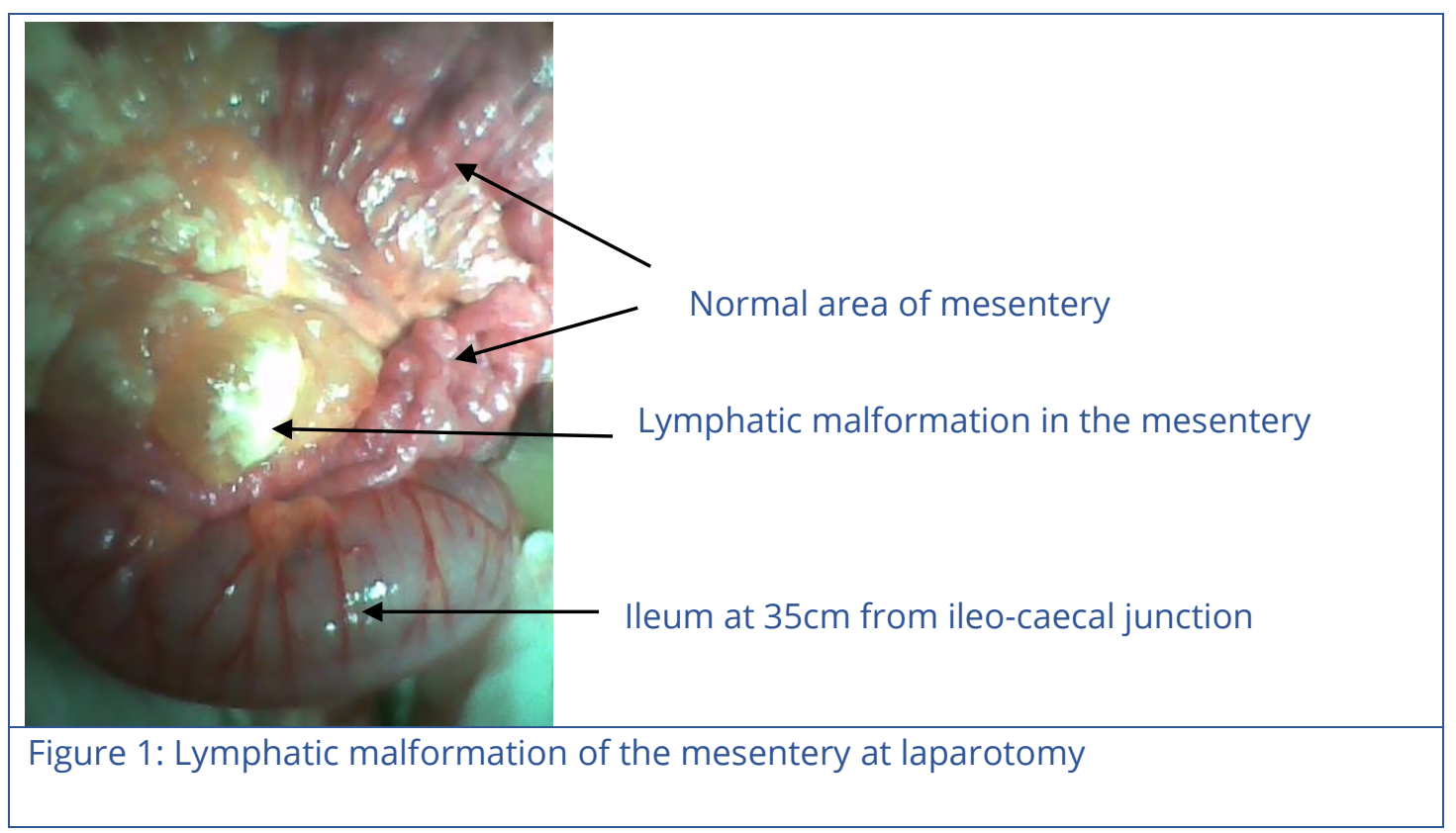

The child made an uneventful recovery. Histology confirmed a lymphatic malformation confined to the resected segment of the mesentery. He remained pain free during subsequent follow up at one-year post surgery.

\section{Discussion}

Management of this child with colicky abdominal pain following meals associated with non-bilious vomiting with normal radiology and inconclusive multidisciplinary expert opinion was a challenge. It is likely that engorgement of the mesenteric lymphatic 
malformation after a heavy meal would have compressed the mesenteric vasculature resulting in an ischaemic type of colicky abdominal pain. The visceral pain induced nonbilious vomiting. The size of the enlarged lymphatics was not large enough to cause obstruction of the small intestinal lumen and induce bilious vomiting.

The upper gastrointestinal contrast studies and contrast CT scans were done after fasting. Fasting would have resulted in collapse of the lymphatic channels making the radiological images appear normal.

Lymphatic malformation of the mesentery causing compression of the mesenteric vasculature without small bowel luminal obstruction is extremely rare and should be considered in children with similar clinical presentations.

\section{References}

1. Ricketts RR: Mesenteric and Omental Cysts. In O'Neil JA, Rowe MI, Grosfeld JL, Fonkalsrud EW, Coran AG, editors. Pediatric Surgery; 1998. pp1269-1275

2. Shironomae T, Kohno M, Yasui Y, Kuwahara T, Satomi M et al. Mesenteric Lymphatic Malformations in Children: Report of Seven Cases. International Surgery. 2017; InPress. https://doi.org/10.9738/INTSURG-D-17-00012.1

3. Kim H, Lee $\mathrm{C}$, Min HS, Jung S, Metrics PX. Clinical features of mesenteric lymphatic malformation in children. Journal of Pediatric surgery. 2015;51(4):582-587. https://doi.org/10.1016/j.jpedsurg.2015.11.021

4. Lloyd DA: Abdominal surgery: general principles of access. In: Spitz L, Coran AG, (eds): Rob \& Smith's Operative Surgery: Pediatric Surgery. Chapman \& Hall Medical London, 1995; 256-264 